\title{
Cerebrospinal fluid area and syringogenesis in Chiari malformation type I
}

\author{
Davis G. Taylor, MD, ${ }^{1}$ Ajay Chatrath, MS, ${ }^{1}$ Panagiotis Mastorakos, MD, PhD, ${ }^{1,2}$ \\ Gabriella Paisan, MD, ${ }^{1}$ Ching-Jen Chen, MD, ${ }^{1}$ Thomas J. Buell, MD, ${ }^{1}$ and John A. Jane Jr., MD${ }^{1}$ \\ 'Department of Neurological Surgery, University of Virginia Health System, Charlottesville, Virginia; and ${ }^{2}$ Surgical Neurology \\ Branch, National Institute of Neurological Disorders and Stroke, National Institutes of Health, Bethesda, Maryland
}

\begin{abstract}
OBJECTIVE Syringogenesis in Chiari malformation type I (CM-I) is thought to occur secondary to impaction of the cerebellar tonsils within the foramen magnum (FM). However, the correlation between the CSF area and syringogenesis has yet to be elucidated. The authors sought to determine whether the diminution in subarachnoid space is associated with syringogenesis. Further, the authors sought to determine if syrinx resolution was associated with the degree of expansion of subarachnoid spaces after surgery.
\end{abstract}

METHODS The authors performed a retrospective review of all patients undergoing posterior fossa decompression for CM-I from 2004 to 2016 at the University of Virginia Health System. The subarachnoid spaces at the FM and at the level of the most severe stenosis were measured before and after surgery by manual delineation of the canal and neural tissue area on MRI and verified through automated CSF intensity measurements. Imaging and clinical outcomes were then compared.

RESULTS Of 68 patients, 26 had a syrinx at presentation. Syrinx patients had significantly less subarachnoid space at the FM (13\% vs 19\%, $p=0.0070)$ compared to those without syrinx. Following matching based on degree of tonsillar herniation and age, the subarachnoid space was significantly smaller in patients with a syrinx (12\% vs $19 \%, p=0.0015)$. Syrinx resolution was associated with an increase in patients' subarachnoid space after surgery compared with those patients without resolution ( $23 \%$ vs $10 \%, p=0.0323)$.

CONCLUSIONS Syrinx development in CM-I patients is correlated with the degree to which the subarachnoid CSF spaces are diminished at the cranial outlet. Successful syrinx reduction is associated with the degree to which the subarachnoid spaces are increased following surgery.

https://thejns.org/doi/abs/10.3171/2019.11.JNS191439

KEYWORDS Chiari I malformation; syringomyelia; posterior fossa decompression; subarachnoid space; skull base

$\mathrm{D}$ EVELOPMENT of syringomyelia is a known occurrence in some, but not all, patients with Chiari malformation type I (CM-I). ${ }^{15}$ Syringogenesis is thought to occur secondary to changes in CSF pathways and the resultant percussion waves generated by the impacted cerebellar tonsils during the cardiac cycle.,3,16 Previous work has shown that patients with smaller posterior fossa anatomy are also more likely to demonstrate abnormalities in CSF and are at increased risk of syringogenesis.,10,13,14,17,20-22 However, the development of syringomyelia in CM-I is not universal, and the association of syrinx development with the CSF area has not yet been fully investigated. Previously, we performed a retrospective analysis of CM-I patients with spacious or crowded posterior fossa subtypes, noting a trend toward syrinx development in crowded subtypes. ${ }^{24}$ At that time, we also noted that the level of most severe stenosis (MSS) in most patients was not at the foramen magnum (FM), but typically occurred slightly more inferiorly, at the level of the 1 st cervical vertebrae $(\mathrm{Cl})$. We hypothesized that this obstruction may alter CSF flow through the cranial outlet and that patients with a syrinx would be more likely to have more severe obstruction of the subarachnoid space. To further investigate this association, we performed a retro-

ABBREVIATIONS CM-I = Chiari malformation type I; FM = foramen magnum; MSS = most severe stenosis; UVA = University of Virginia Health System. SUBMITTED June 9, 2019. ACCEPTED November 26, 2019.

INCLUDE WHEN CITING Published online February 21, 2020; DOI: 10.3171/2019.11.JNS191439. 

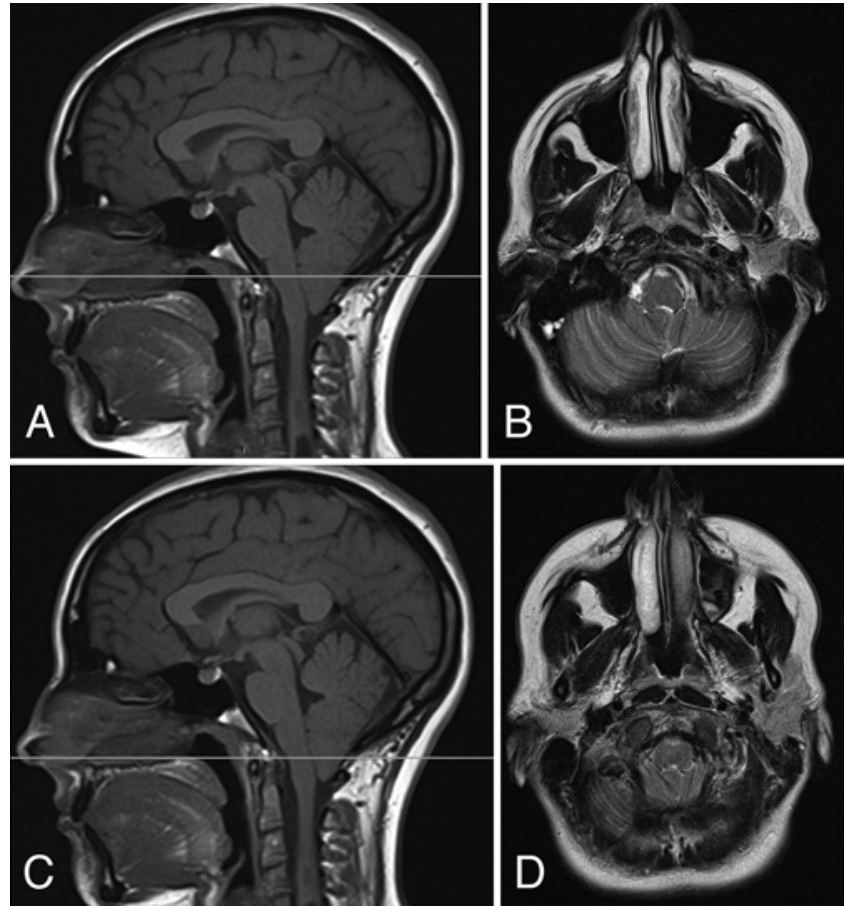

FIG. 1. Images at the FM and at the MSS, which was typically just inferior to the odontoid tip. Midline sagittal T1-weighted MRI was used to identify the levels of the FM (A) and MSS (C). Axial T2-weighted MRI cross sections are shown at the levels of the FM (B) and MSS (D)

spective, matched cohort study involving detailed imaging analysis of CM-I patients with and those without a syrinx who were treated at our institution.

\section{Methods}

\section{Patients}

After obtaining institutional review board approval, we retrospectively evaluated patients who underwent posterior fossa decompression for CM-I during the period from November 2004 to December 2016. All surgeries were performed by 2 attending neurosurgeons (J.A.J. and Edward H. Oldfield) at the University of Virginia Health System (UVA). Inclusion criteria required a diagnosis of
CM-I with at least 5-mm tonsillar herniation on midline sagittal T1-weighted MRI, undergoing posterior fossa decompression, patient age 5 years or older, and preoperative T1-weighted sagittal and T2-weighted axial MR images available for review.

\section{Radiographic Analysis of CSF Area}

All patients underwent routine preoperative T1- and T2weighted MRI in the standard supine position. Radiology reports were then reviewed for diagnostic impression, including the presence or absence of a syrinx. Syrinx presence was determined by independent radiologists as noted on the preoperative MRI report. Expansion of the cord at the level of the syrinx was required to differentiate a syrinx from hydromyelia. The minimum syrinx width was 5.1 $\mathrm{mm}$. The mean syrinx width was $8.0 \pm 2.2 \mathrm{~mm}$. All syrinxes were located in the cervical spine. All patients underwent preoperative T1- and T2-weighted MRI. Cine MRI studies were obtained in 52 of the 68 patients included in this study ( 34 from patients with a syrinx and 18 from patients without a syrinx), and interpretations of the radiological studies were reviewed for documentation of normal or abnormal flow and diagnostic impression of CM-I.

The images were then reviewed by 2 of the authors (A.C. and G.P.) using a picture archiving and communication system (Carestream Health, Inc.). The authors were not blinded to the study objectives. To evaluate CSF area, T2-weighted axial images were reviewed and the canal area (measured as the boundary of hypointense ligament or dura surrounding hyperintense CSF) and intraparenchymal area (measured as the area of the isointense intraparenchymal component within the CSF area at the same level) were manually delineated, and the area of each was computed with Carestream Health software. Axial images were selected at the FM and again at the MSS, typically just inferior to the level of the odontoid tip (Fig. 1). The difference between the canal and tissue areas was then calculated and used as a representation of CSF area at the level of MSS (Fig. 2).

Because voxel acquisition may lead to some discrepancy in tissue planes on MRI, automated corrected integrated density was utilized to confirm the accuracy of manual CSF area measurements. The previously evaluated T2-weighted axial slices were imported into open-
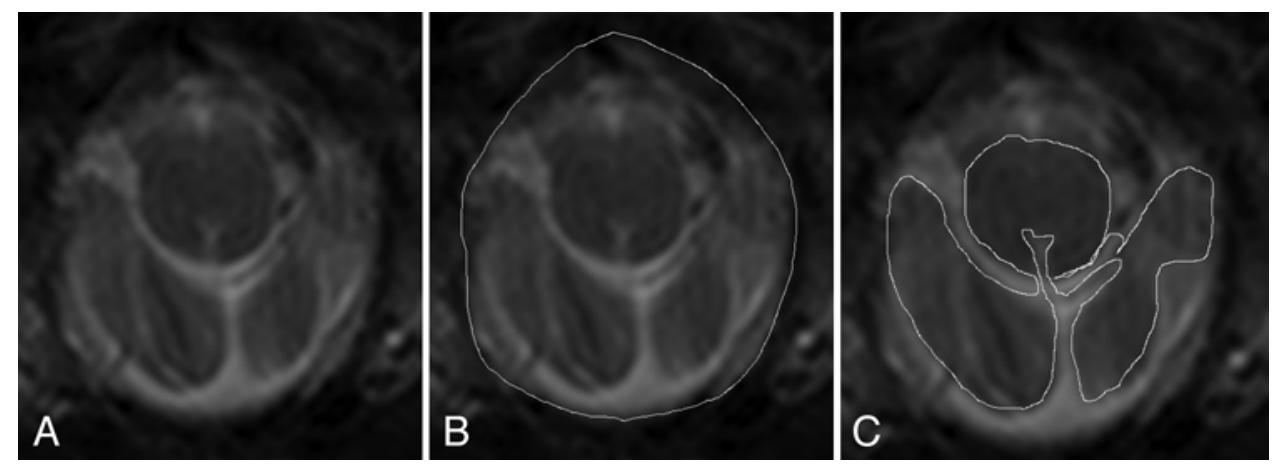

FIG. 2. An axial cross section at the level of the FM or MSS (A) was used to quantify the CSF area by subtracting the area of the canal (B) and the area of the tissue (C). 
source ImageJ $(\mathrm{NIH})$ software. ${ }^{18}$ The region of interest was again manually delineated to include the spinal canal at the previously determined level of MSS. The selected area was then analyzed by ImageJ to determine the corrected integrated density and mean gray value at the region of interest. Background control samples were recorded by acquisition of multiple regions of interest at the medulla or spinal cord to correct for variations in MRI signals between patients, and a corrected integrated density value was calculated by ImageJ. Similar methods have been previously described. ${ }^{8}$

In patients with syringomyelia, measurements similar to those performed on preoperative images were repeated on follow-up postoperative MR images. The new CSF area was recorded and the change in area was computed. Resolution, improvement, or persistence of syringomyelia was documented according to determinations recorded on the official radiography reports. In patients who did not experience resolution of their syrinx, imaging was reviewed and interpreted to determine any causative factors.

\section{Surgical Procedure}

All patients at UVA underwent standardized decompression of the posterior fossa performed by one of 2 attending neurosurgeons. In general, the posterior arch of $\mathrm{C} 1$ and the suboccipital bone were removed to a standard width of $2.5 \mathrm{~cm}$ (range $2.0-4.0 \mathrm{~cm}$ ) and the suboccipital craniectomy was extended to a standard height of $2 \mathrm{~cm}$ (range $0.70-2.50 \mathrm{~cm}$ ), although there was some variability based on surgeon discretion.

All operative notes were reviewed by one of the authors (A.C. or G.P.) for detailed specifics of the procedure, including whether a pericranial graft or dural graft was used and whether microsurgical lysis of adhesions or cautery of cerebellar tonsils was performed. In 14 patients, the craniotomy defect dimensions were not specifically mentioned in the operative note, so postoperative $\mathrm{CT}$ was used to measure the defect size instead.

\section{Statistical Methods}

The Mann-Whitney test was used to determine statistical differences in the area of CSF space between syrinx and nonsyrinx patients. Chi-square analysis was used to determine statistical differences in categorical values. A p value less than 0.05 was considered significant.

Because we observed a significant difference in age at the time of treatment and degree of tonsillar herniation on midline sagittal MRI between patients with and those without a syrinx, we repeated all statistical tests after one-to-one matching of patients with a syrinx to patients without a syrinx. The 2 cohorts were matched, without replacement, in a 1:1 ratio with a caliper of 0.2 using propensity scores derived from covariates of age and degree of tonsillar herniation. The matching was performed using the PSMATCH2 package developed for Stata (version 14.2). ${ }^{7}$

\section{Results}

In total, 69 patients who were diagnosed with CM-I and treated at UVA between 2004 and 2016 met the initial
TABLE 1. Demographic, clinical, and surgical data among all CM-I patients

\begin{tabular}{lcccc}
\hline & All & No Syrinx & Syrinx & p Value \\
\hline No. of patients & 68 & 42 & 26 & \\
\hline Female & $66 \%$ & $64 \%$ & $68 \%$ & 0.2446 \\
\hline Age, yrs & 28.09 & 32.37 & 21.17 & 0.0071 \\
\hline Tonsillar herniation, mm & 13.55 & 12.24 & 15.62 & 0.0360 \\
\hline Cervicomedullary kinking & $38 \%$ & $33 \%$ & $46 \%$ & 0.2904 \\
\hline Abnormal cine & $88 \%$ & $85 \%$ & $94 \%$ & 0.3258 \\
\hline Symptomatic & $94 \%$ & $100 \%$ & $88 \%$ & 0.0353 \\
\hline Headache & $86 \%$ & $85 \%$ & $87 \%$ & 0.8606 \\
\hline Symptom resolution & $75 \%$ & $76 \%$ & $74 \%$ & 0.8804 \\
\hline Defect height, mm & 2.09 & 2.09 & 2.08 & 0.9577 \\
\hline Defect width, mm & 2.62 & 2.56 & 2.72 & 0.0924 \\
\hline Duroplasty & $57 \%$ & $64 \%$ & $46 \%$ & 0.1418 \\
\hline Dural splitting & $41 \%$ & $33 \%$ & $54 \%$ & 0.0949 \\
\hline Lysis of adhesions & $13 \%$ & $12 \%$ & $15 \%$ & 0.6807 \\
\hline Tonsil cautery & $12 \%$ & $10 \%$ & $15 \%$ & 0.4660 \\
\hline Patents with syinx & & &
\end{tabular}

Patients with syrinx presented at a younger age (21.17 vs 32.37 years, $p=$ 0.0071 ) and with more severe tonsillar herniation (15.62 vs $12.24 \mathrm{~mm}, p=$ 0.0360 ) than patients without syrinx. Boldface type indicates statistical significance.

inclusion criteria. One patient was excluded due to insufficient preoperative imaging, meaning clinical data and outcomes from 68 patients were included in this study. Of these 68 patients, 26 presented with a syrinx and 42 presented without a syrinx. Clinical characteristics for these patients are presented in Table 1 . There were no differences in the demographic, clinical, or surgical characteristics between patients presenting with and those presenting without a syrinx, other than age at time of treatment (21.2 vs 32.4 years, $p=0.0071$ ) and degree of tonsillar herniation on midline sagittal MRI (15.62 vs $12.24 \mathrm{~mm}$, $\mathrm{p}=0.0360)$.

Imaging characteristics among patients with and those without a syrinx are presented in Table 2. Patients with syringomyelia had a significantly decreased CSF area at the FM compared with patients without syringomyelia (area decreased $13 \%$ vs $19 \%$, respectively, $\mathrm{p}=0.0070$ ) and at the level of MSS ( $4 \%$ vs $10 \%, p=0.0026$ ), based on manually delineated CSF areas. For integrated density verified through automated CSF signal intensity measures, patients with syrinx had a significantly lower corrected integrated density (less CSF signal) at the FM (24.10 vs 32.12 , $\mathrm{p}=0.0102)$ and at the MSS (13.90 vs 19.29, $\mathrm{p}=0.0187$ ) based on automated computation, corresponding with our manual measurements.

Due to the statistical differences in patient demographics, one-to-one matching of nonsyrinx to syrinx patients based on degree of tonsillar herniation and age was performed. After matching, 22 patients were included in each group. Clinical and demographic characteristics of the matched cohorts are presented in Table 3. There were no statistical differences between groups. Imaging characteristics for the matched cohorts are presented in Table 4. Following matching, there was significantly decreased 
TABLE 2. Imaging characteristics of all CM-I patients at the levels of the FM and MSS

\begin{tabular}{lrrrr}
\hline \multicolumn{1}{c}{ Imaging } & All & No Syrinx & Syrinx & p Value \\
\hline Preop & & & & \\
\hline MSS at C1 & $72 \%$ & $76 \%$ & $65 \%$ & 0.3345 \\
\hline CSF area at FM & $16 \%$ & $19 \%$ & $13 \%$ & 0.0070 \\
\hline CSF area at MSS & $8 \%$ & $10 \%$ & $4 \%$ & 0.0026 \\
\hline CSF signal at FM & 29.05 & 32.12 & 24.10 & 0.0102 \\
\hline CSF signal at MSS & 17.23 & 19.29 & 13.90 & 0.0187 \\
\hline Follow-up & & & & \\
\hline CSF area at FM & $37 \%$ & $38 \%$ & $34 \%$ & 0.1720 \\
\hline CSF area at MSS & $28 \%$ & $30 \%$ & $25 \%$ & 0.0727 \\
\hline CSF signal at FM & 52.28 & 54.53 & 48.97 & 0.1637 \\
\hline CSF signal at MSS & 19.97 & 21.77 & 17.46 & 0.0817 \\
\hline CSF area change at FM & $21 \%$ & $20 \%$ & $21 \%$ & 0.6952 \\
\hline CSF area change at MSS & $21 \%$ & $21 \%$ & $21 \%$ & 0.9422 \\
\hline
\end{tabular}

Patients with syrinx demonstrated more severe CSF obstruction at the FM ( $13 \%$ vs $19 \%$ canal area patency, $p=0.0070$ ). In some patients, patency is most restricted below the FM, resulting in more severe obstruction of CSF pathways ( $4 \%$ vs $10 \%$ patency, $p=0.026$ ). These findings are supported by automated CSF signal intensity measurements (CSF signal at FM and MSS). Boldface type indicates statistical significance.

CSF area in syrinx patients compared with nonsyrinx patients at both the FM $(12 \%$ vs $19 \%, \mathrm{p}=0.0015)$ and the MSS (5\% vs 9\%, $\mathrm{p}=0.0454)$ based on the manually delineated CSF area. Compared with nonsyrinx patients, patients with a syrinx also had lower corrected integrated density (reduced CSF signal) at the FM (23.57 vs 32.17, $\mathrm{p}=0.0112)$ and at the MSS (13.63 vs 17.92, $\mathrm{p}=0.1244)$,

TABLE 3. Matched cohort comparison between patients with and those without syrinx

\begin{tabular}{lcccc}
\hline & All & No Syrinx & Syrinx & p Value \\
\hline No. of patients & 44 & 22 & 22 & \\
\hline Female & $66 \%$ & $64 \%$ & $68 \%$ & 0.7505 \\
\hline Age, yrs & 22.59 & 23.36 & 21.82 & 0.7464 \\
\hline Tonsillar herniation, mm & 14.05 & 14.86 & 13.23 & 0.3040 \\
\hline Cervicomedullary kinking & $48 \%$ & $45 \%$ & $50 \%$ & 0.7628 \\
\hline Abnormal cine & $88 \%$ & $83 \%$ & $93 \%$ & 0.3808 \\
\hline Symptomatic & $93 \%$ & $100 \%$ & $86 \%$ & 0.0728 \\
\hline Headache & $83 \%$ & $81 \%$ & $84 \%$ & 0.7865 \\
\hline Symptom resolution & $78 \%$ & $81 \%$ & $74 \%$ & 0.5825 \\
\hline Defect height, mm & 1.99 & 1.95 & 2.03 & 0.5404 \\
\hline Defect width, mm & 2.65 & 2.59 & 2.72 & 0.3193 \\
\hline Duroplasty & $52 \%$ & $59 \%$ & $45 \%$ & 0.3652 \\
\hline Dural splitting & $45 \%$ & $36 \%$ & $55 \%$ & 0.2259 \\
\hline Lysis of adhesions & $18 \%$ & $23 \%$ & $14 \%$ & 0.4344 \\
\hline Tonsil cautery & $16 \%$ & $18 \%$ & $14 \%$ & 0.6802 \\
\hline
\end{tabular}

Patients were controlled for age and severity of tonsillar herniation.
TABLE 4. Comparison of imaging characteristics at the levels of the FM and MSS in age- and tonsillar herniation-matched CM-I patients

\begin{tabular}{lrrrr}
\hline \multicolumn{1}{c}{ Imaging } & All & No Syrinx & Syrinx & p Value \\
\hline Preop & & & & \\
\hline MSS at C1 & $66 \%$ & $73 \%$ & $59 \%$ & 0.3400 \\
\hline CSF area at FM & $16 \%$ & $19 \%$ & $12 \%$ & 0.0015 \\
\hline CSF area at MSS & $7 \%$ & $9 \%$ & $5 \%$ & 0.0454 \\
\hline CSF signal at FM & 27.87 & 32.17 & 23.57 & 0.0112 \\
\hline CSF signal at MSS & 15.77 & 17.92 & 13.63 & 0.1244 \\
\hline Follow-up & & & & \\
\hline CSF area at FM & $37 \%$ & $37 \%$ & $38 \%$ & 0.8899 \\
\hline CSF area at MSS & $30 \%$ & $29 \%$ & $30 \%$ & 0.7999 \\
\hline CSF signal at FM & 52.18 & 53.3 & 51.06 & 0.6716 \\
\hline CSF signal at MSS & 19.68 & 20.83 & 18.54 & 0.4935 \\
\hline CSF area change at FM & $22 \%$ & $18 \%$ & $25 \%$ & 0.0490 \\
\hline CSF area change at MSS & $22 \%$ & $22 \%$ & $22 \%$ & 0.9800 \\
\hline
\end{tabular}

Analysis demonstrates more severe CSF obstruction at the FM ( $12 \%$ vs $19 \%$, $p=0.0015)$. Obstruction was worse in some patients below the FM, leading to further reduction in CSF patency ( $5 \%$ vs $9 \%, p=0.0454)$. Patients with syrinx experienced a greater improvement in CSF patency at the FM than patients without syrinx $(25 \%$ vs $18 \%, p=0.049)$. Boldface type indicates statistical significance.

though the decrease at the MSS did not reach statistical significance. At follow-up, patients with a syrinx had a greater change in the CSF area at the FM compared with patients without a syrinx $(25 \%$ vs $18 \%, \mathrm{p}=0.0490)$. Given that at follow-up the patients with and those without a syrinx had similar CSF areas at the FM (38\% vs $37 \%$, p $=0.8899$ ), this difference is primarily reflective of syrinx patients having a lower CSF area initially.

Among the 26 patients with syrinx, 5 did not achieve resolution postoperatively. There were no demographic, clinical, or surgical differences between the patients with syrinx who did and those who did not achieve resolution postoperatively (Table 5). At follow-up, patients with syrinx resolution had a significantly greater change in the CSF area at the level of MSS than patients without syrinx resolution ( $23 \%$ vs $10 \%, p=0.0323$, Table 6). Among patients who did not have syrinx resolution, 2 patients developed a pseudomeningocele and arachnoid adhesions that restricted cerebellar relaxation, and 2 patients had a syrinx that continued to be confined by bony elements. The bony dimensions of the craniotomy in these patients were 2.0 $\mathrm{cm}$ in height for all patients and $2.50-3.0 \mathrm{~cm}$ in width, which were not different from those in patients with syrinx resolution. In one of the patients who had adhesions, a duroplasty was performed and the tonsils were cauterized during surgery. The adhesions were not appreciable before surgery.

\section{Discussion}

CM-I is a neurosurgical pathology resulting from herniation of the cerebellar tonsils into the FM. ${ }^{15}$ Current evidence suggests that CM-I is likely an acquired disease in 
TABLE 5. Demographic data among CM-I patients with syrinx

\begin{tabular}{lcccc}
\hline & All & No Resolution & Improved & p Value \\
\hline No. of patients & 26 & 5 & 21 & \\
\hline Female & $58 \%$ & $100 \%$ & $62 \%$ & 0.6196 \\
\hline Age, yrs & 21.17 & 19.38 & 21.59 & 0.3821 \\
\hline Tonsillar herniation, mm & 15.62 & 15.63 & 15.95 & 0.6861 \\
\hline Cervicomedullary kinking & $46 \%$ & $40 \%$ & $52 \%$ & 0.3636 \\
\hline Abnormal cine & $94 \%$ & $67 \%$ & $100 \%$ & 0.2982 \\
\hline Symptomatic & $88 \%$ & $100 \%$ & $86 \%$ & $>0.999$ \\
\hline Headache & $87 \%$ & $40 \%$ & $83 \%$ & 0.2981 \\
\hline Symptom resolution & $74 \%$ & $0 \%$ & $67 \%$ & 0.2725 \\
\hline Defect height, mm & 2.08 & 2.00 & 2.10 & 0.3739 \\
\hline Defect width, mm & 2.72 & 2.75 & 2.71 & 0.3739 \\
\hline Duroplasty & $46 \%$ & $60 \%$ & $48 \%$ & $>0.999$ \\
\hline Dural splitting & $54 \%$ & $20 \%$ & $52 \%$ & $>0.999$ \\
\hline Lysis of adhesions & $15 \%$ & $20 \%$ & $14 \%$ & $>0.999$ \\
\hline Tonsil cautery & $15 \%$ & $20 \%$ & $14 \%$ & $>0.999$ \\
\hline
\end{tabular}

There was no association between age, tonsillar herniation, symptoms, or surgical technique and resolution of syrinx.

individuals with insufficient bony growth, though it can also present secondary to another disease process. ${ }^{2,6,11-13,23}$ The pathological hallmark of CM-I is impaction of the cerebellar tonsils within the FM. ${ }^{4}$ Previous studies have demonstrated that the impaction of the cerebellar tonsils into the cervical canal results in a "water hammer" effect in concert with the cardiac cycle that is transmitted to the cervical canal. ${ }^{3,15,16}$ On the basis of this finding, it was proposed that the percussive fluid waves created during systole propagate through the CSF along Virchow-Robbin spaces, resulting in increased interstitial pressure within the cervical cord and consequent syringomyelia development.,15

Morphologically, it has been previously demonstrated that patients with preserved CSF space anterior to the cord are less likely to be symptomatic at presentation than those with decreased ventral CSF space, and symptomatic patients with normal CSF flow are less likely to respond to posterior fossa decompression than those with altered CSF flow dynamics on cine-phase MRI. ${ }^{5,9}$ These findings suggest that CSF dynamics play a role in symptom response following surgery. The relationship between syringogenesis and obstruction of CSF pathways has also been investigated, and prior studies have found that patients with crowded posterior fossa subtypes are more likely to experience syrinx formation than those without obvious posterior fossa crowdedness. ${ }^{19,24}$ Still, not all patients with CM-I develop a syrinx, and not all patients clinically respond to posterior fossa decompression. Therefore, understanding the pathophysiology of syringogenesis is critical to determining clinical response to surgery, and determination of what variables may promote a response to surgical therapy is essential to improving patient outcomes. The association with CSF area has not been previously established.

Our findings demonstrate that patients with a syrinx are significantly more likely to have smaller CSF areas than those without a syrinx, as confirmed either by imaging in-
TABLE 6. Imaging characteristics among all CM-I patients with syrinx at the level of the FM and MSS

\begin{tabular}{lrrrr}
\hline \multicolumn{1}{c}{ Imaging } & All & No Resolution & Improved & p Value \\
\hline Preop & & & & \\
\hline MSS at C1 & $65 \%$ & $60 \%$ & $71 \%$ & 0.3022 \\
\hline CSF area at FM & $13 \%$ & $11 \%$ & $14 \%$ & 0.3754 \\
\hline CSF area at MSS & $4 \%$ & $6 \%$ & $4 \%$ & 0.8525 \\
\hline CSF signal at FM & 24.10 & 22.93 & 24.37 & 0.56 \\
\hline CSF signal at MSS & 13.90 & 16.23 & 13.35 & 0.6737 \\
\hline Follow-up & & & & \\
\hline CSF area at FM & $34 \%$ & $24 \%$ & $36 \%$ & 0.1017 \\
\hline CSF area at MSS & $25 \%$ & $15 \%$ & $28 \%$ & 0.0670 \\
\hline CSF signal at FM & 48.97 & 35.27 & 52.23 & 0.1018 \\
\hline CSF signal at MSS & 17.46 & 9.96 & 19.25 & 0.0774 \\
\hline CSF area change at FM & $21 \%$ & $14 \%$ & $23 \%$ & 0.1397 \\
\hline CSF area change at MSS & $21 \%$ & $10 \%$ & $23 \%$ & 0.0323 \\
\hline
\end{tabular}

There were no differences in CSF patency at presentation among those patients with and those without syrinx resolution. Syrinx resolution was associated with a greater restoration of CSF patency at the level of MSS ( $23 \%$ vs $10 \%, p=0.0323)$. Boldface type indicates statistical significance.

terpretation or through automated CSF signal determination. Severity of canal crowdedness in these patients was not only visually apparent but also supported by both manual and automated measurements. Syrinx resolution, as determined by expansion of the CSF space at the level of MSS, was correlated with syrinx improvement at the MSS rather than at the FM. Interestingly, among those patients who did not improve, 1 patient did not have any abnormality on cine flow, whereas all patients with syrinx improvement had abnormal cine studies. Our results suggest that disruption of CSF flow is significantly associated with syringogenesis and indeed may be the instigating culprit.

\section{Study Limitations}

Our study is limited by the retrospective design and reliance on imaging interpretation, which inherently lends itself to internal bias. Automated intensity quantification was used to reduce bias, and the findings agreed with our manual interpretation of CSF spaces. Syrinx formation and expansion of the cord typically occur within the cervical spine, well below the cranial outlet. However, if the syrinx does expand into the FM it could result in cord expansion and subsequently a decrease in the area of CSF space, which may have influenced our measurements of the CSF area. In this study, we analyzed single axial slices as a correlate to patients' 3D anatomy, although in the future evaluation of multiple slices may be more accurate. Other limitations include the selection bias inherent in noncontrolled surgical studies and the limited statistical power to detect significant differences resulting from the relatively modest number of patients included in this study.

\section{Conclusions}

Syringogenesis in patients with CM-I is an indication 
for surgical intervention to prevent syrinx expansion and subsequent spinal cord damage. The cause of syringogenesis is not yet fully understood. In this study, we demonstrated that compared with patients without a syrinx, patients with a syrinx have significantly decreased patency of subarachnoid spaces and that restoration of subarachnoid patency is associated with syrinx resolution. Further research is needed to understand the treatment for CM-I in patients with syringomyelia, as well as the pathogenesis of syringomyelia in patients without obstruction of the subarachnoid space.

\section{References}

1. Atkinson JL, Kokmen E, Miller GM: Evidence of posterior fossa hypoplasia in the familial variant of adult Chiari I malformation: case report. Neurosurgery 42:401-404, 1998

2. Furtado SV, Reddy K, Hegde AS: Posterior fossa morphometry in symptomatic pediatric and adult Chiari I malformation. J Clin Neurosci 16:1449-1454, 2009

3. Heiss JD, Patronas N, DeVroom HL, Shawker T, Ennis R, Kammerer W, et al: Elucidating the pathophysiology of syringomyelia. J Neurosurg 91:553-562, 1999

4. Heiss JD, Suffredini G, Bakhtian KD, Sarntinoranont M, Oldfield EH: Normalization of hindbrain morphology after decompression of Chiari malformation Type I. J Neurosurg 117:942-946, 2012

5. Ishikawa M, Kikuchi H, Fujisawa I, Yonekawa Y: Tonsillar herniation on magnetic resonance imaging. Neurosurgery 22:77-81, 1988

6. Kasner SE, Rosenfeld J, Farber RE: Spontaneous intracranial hypotension: headache with a reversible Arnold-Chiari malformation. Headache 35:557-559, 1995

7. Leuven E, Sianesi B: PSMATCH2: Stata module to perform full Mahalanobis and propensity score matching, common support graphing, and covariate imbalance testing. EconPapers (http://EconPapers.repec.org/ RePEc:boc:bocode:s432001) [Accessed December 13, 2019]

8. McCloy RA, Rogers S, Caldon CE, Lorca T, Castro A, Burgess A: Partial inhibition of Cdk1 in $\mathrm{G} 2$ phase overrides the SAC and decouples mitotic events. Cell Cycle 13:1400-1412, 2014

9. McGirt MJ, Nimjee SM, Fuchs HE, George TM: Relationship of cine phase-contrast magnetic resonance imaging with outcome after decompression for Chiari I malformations. Neurosurgery 59:140-146, 2006

10. Milhorat TH, Chou MW, Trinidad EM, Kula RW, Mandell $\mathrm{M}$, Wolpert C, et al: Chiari I malformation redefined: clinical and radiographic findings for 364 symptomatic patients. Neurosurgery 44:1005-1017, 1999

11. Milhorat TH, Nishikawa M, Kula RW, Dlugacz YD: Mechanisms of cerebellar tonsil herniation in patients with Chiari malformations as guide to clinical management. Acta Neurochir (Wien) 152:1117-1127, 2010

12. Morioka T, Shono T, Nishio S, Yoshida K, Hasuo K, Fukui M: Acquired Chiari I malformation and syringomyelia associated with bilateral chronic subdural hematoma. Case report. J Neurosurg 83:556-558, 1995

13. Nishikawa M, Sakamoto H, Hakuba A, Nakanishi N, Inoue Y: Pathogenesis of Chiari malformation: a morphometric study of the posterior cranial fossa. J Neurosurg 86:40-47, 1997
14. Nyland H, Krogness KG: Size of posterior fossa in Chiari type 1 malformation in adults. Acta Neurochir (Wien) 40:233-242, 1978

15. Oldfield EH: Pathogenesis of Chiari I-pathophysiology of syringomyelia: implications for therapy: a summary of 3 decades of clinical research. Neurosurgery 64 (CN Suppl 1):66-77, 2017

16. Oldfield EH, Muraszko K, Shawker TH, Patronas NJ: Pathophysiology of syringomyelia associated with Chiari I malformation of the cerebellar tonsils. Implications for diagnosis and treatment. J Neurosurg 80:3-15, 1994

17. Pinna G, Alessandrini F, Alfieri A, Rossi M, Bricolo A: Cerebrospinal fluid flow dynamics study in Chiari I malformation: implications for syrinx formation. Neurosurg Focus 8(3):E3, 2000

18. Schindelin J, Arganda-Carreras I, Frise E, Kaynig V, Longair M, Pietzsch T, et al: Fiji: an open-source platform for biological-image analysis. Nat Methods 9:676-682, 2012

19. Sgouros S, Kountouri M, Natarajan K: Posterior fossa volume in children with Chiari malformation Type I. J Neurosurg 105 (2 Suppl):101-106, 2006

20. Shah S, Haughton V, del Río AM: CSF flow through the upper cervical spinal canal in Chiari I malformation. AJNR Am J Neuroradiol 32:1149-1153, 2011

21. Støverud KH, Langtangen HP, Ringstad GA, Eide PK, Mardal KA: Computational investigation of cerebrospinal fluid dynamics in the posterior cranial fossa and cervical subarachnoid space in patients with Chiari I malformation. PLoS One 11:e0162938, 2016

22. Stovner LJ, Bergan U, Nilsen G, Sjaastad O: Posterior cranial fossa dimensions in the Chiari I malformation: relation to pathogenesis and clinical presentation. Neuroradiology 35:113-118, 1993

23. Tachibana S, Harada K, Abe T, Yamada H, Yokota A: Syringomyelia secondary to tonsillar herniation caused by posterior fossa tumors. Surg Neurol 43:470-477, 1995

24. Taylor DG, Mastorakos P, Jane JA Jr, Oldfield EH: Two distinct populations of Chiari I malformation based on presence or absence of posterior fossa crowdedness on magnetic resonance imaging. J Neurosurg 126:1934-1940, 2017

\section{Disclosures}

The authors report no conflict of interest concerning the materials or methods used in this study or the findings specified in this paper.

\section{Author Contributions}

Conception and design: Taylor. Acquisition of data: Taylor, Chatrath, Paisan. Analysis and interpretation of data: all authors. Drafting the article: all authors. Critically revising the article: all authors. Reviewed submitted version of manuscript: all authors. Approved the final version of the manuscript on behalf of all authors: Taylor. Statistical analysis: Taylor, Chatrath, Paisan, Chen, Buell. Administrative/technical/material support: Jane. Study supervision: Mastorakos, Jane.

\section{Correspondence}

Davis G. Taylor: University of Virginia, Charlottesville, VA. dt4aa@virginia.edu. 